\title{
Effectiveness and safety of bovine pericardium patch repair for cervical anastomotic leakage after oesophagectomy for cancer
}

\author{
Xionghuai Hua ${ }^{1}$, Rulin Qian ${ }^{1}$, Kefeng Shi ${ }^{1}$, Xiufeng Wei ${ }^{2}$, Heng Zhang ${ }^{1}$, Ge Qu ${ }^{1}$, Maolin Chen $^{1}$, \\ Binbin Zhang ${ }^{1}$ \\ ${ }^{1}$ Department of Thoracic Surgery, Henan Chest Hospital, Zhengzhou 450000, China; ${ }^{2}$ Department of Thoracic Surgery, the First Affiliated Hospital \\ of Xinxiang Medical University, Weihui 453100, China \\ Contributions: (I) Conception and design: X Hua; (II) Administrative support: R Qian, K Shi; (III) Provision of study materials or patients: H Zhang, \\ M Chen, X Wei, G Qu, B Zhang; (IV) Collection and assembly of data: X Hua, X Wei; (V) Data analysis and interpretation: X Hua, X Wei; (VI) \\ Manuscript writing: All authors; (VII) Final approval of manuscript: All authors. \\ Correspondence to: Rulin Qian. Department of Thoracic Surgery, Henan Chest Hospital, Zhengzhou 450000, China. Email: xwnj2012@163.com.
}

\begin{abstract}
Background: The objective of this study is to evaluate the effectiveness and safety of bovine pericardium patch (BPP) repair for cervical anastomotic leakage after esophageal squamous cancer.

Methods: Intractable cervical anastomotic leakage developed in 7 patients of esophageal squamous cell carcinoma undergoing cervical anastomosis. These patients received the BPP repair. The necrotic tissue around the cervical anastomosis was removed during the operation, and the defect was repaired with BPP according to the size of the leakage.

Results: The operative duration was 60-90 min (median, $75 \mathrm{~min}$ ). There were no signs of recurrent anastomotic leakage in each patient undergoing BPP repair. Oral intake was initiated 5-8 days (median, 6 days) after the BPP repair operation without any discomfort.

Conclusions: The BPP repair is a safe and effective processing scheme for patients with cervical anastomotic fistula after resection of esophageal squamous cell carcinoma. This method may be recommended for appropriate patients with intractable cervical anastomotic fistula.
\end{abstract}

Keywords: Esophageal squamous cancer; esophageal reconstruction; cervical anastomotic leakage; bovine pericardium patch (BPP)

Submitted Aug 27, 2019. Accepted for publication Sep 04, 2019.

doi: $10.21037 /$ jtd.2019.09.12

View this article at: http://dx.doi.org/10.21037/jtd.2019.09.12

\section{Introduction}

Resection of esophageal carcinoma is one of the surgical operations with the complex operation and high incidence of complications. In clinic, the conventional surgical methods of resection and digestive tract reconstruction of esophageal carcinoma are as follows: Ivor-Lewis esophagectomy, the McKeown approach, and the trans hiatal technique. Especially, minimally invasive resection of the esophagus is becoming increasingly popular in recent years (1). In China, thoracic-laparoscopic esophagectomy is the current mainstream. Regardless of the technique, the residual stomach is the most commonly used replacement organ after esophageal reconstruction (2). Cervical esophagogastric anastomotic fistula is one of the most common complications after esophageal squamous cancer. It may prolong the hospital stay, and cause severe mediastinal infections, even death in a short time (3).

Adequate drainage and active anti-infection treatment in the early stage are the key measures to control the further deterioration of cervical anastomotic leakage (4). Besides, it is necessary to correct the malnourishment. After a period of conservative treatment, if the fistula does not improve, further surgical or interventional intervention should be considered $(5,6)$. In this study, we tried to use bovine pericardium patch (BPP) to repair cervical anastomotic 
leakage after esophagectomy for cancer in order to evaluate its safety and effectiveness.

\section{Methods}

One hundred sixty-two patients with esophageal squamous cell carcinoma underwent radical resection at the Department of Thoracic Surgery, Henan Chest Hospital from June 2017 to June 2019. Among them, 142 patients underwent tubular gastric reconstruction with cervical esophagogastric anastomosis. The thoracoscopy combined laparoscopy and mediastinoscopy combined with laparoscopy surgical treatment were performed in 112 and 30 patients, respectively.

The end-to-side esophagogastric cervical anastomosis was performed in all patients by mechanical anastomosis by a 25-mm-diameter circular stapler. After the gastric stump was cut into a tubular gastric outside the abdominal cavity, it was pulled up to the neck along the posterior mediastinal esophageal bed. The distal end of the tubular gastric was cut, the circular stapler was inserted, and the gastric wall was penetrated. Then the center rod of the circular stapler was matched with the anvil which has been placed into the stump of the cervical esophagus. The gastric stump incision was closed with the linear cutting-closure device. Finally, the cervical anastomosis was intermittent and strengthened with 4-0 silk thread.

Anastomotic leakage was diagnosed in this study by salivary discharge, esophagography, or endoscopy. Cervical anastomotic leakage developed in 18 patients $(12.7 \%$ ) with esophageal squamous cell carcinoma undergoing esophagogastric cervical anastomosis. In these patients, 11 patients cured within 6 weeks by conservative treatment, including anti-infective therapy and adequate drainage. Finally, 7 patients developed into persistent and intractable anastomotic leakage. All of these patients with intractable anastomotic leakage were confirmed by routine endoscopic examination or angiography.

All patients were needed to sign an informed consent form before the operation. Moreover, all the BPP repair operation was performed under general anesthesia. The repair process of BPP mainly included two aspects. That was debridement of the leakage and repair of defect with BPP. The necrotic tissue around the leakage was removed, and the region of esophagogastric anastomosis leakage was exposed. Then according to the size of fistula, the appropriate BPP was made. Repair of the esophagogastric anastomosis defect was performed with continuous sutures using 3-0 slide suture (Prolene W8558; Ethicon Inc.). Finally, a closed drain was inserted near the BPP (Figure 1).

\section{Results}

The clinical features of these 7 patients who underwent BPP repair were summarized (Table 1 ). Five of them were male $(71.4 \%)$. The average age was 66.9 years (range, 55-78 years). Based on the stage of esophageal squamous cell carcinoma (TNM classification, 8th edition, UICC), patient classification was as follows: stage IB, 2 patients; stage IIA, 2 patients; stage IIIA, 2 patients; and stage IIIB, 1 patient. Gastric conduit reconstruction was performed in all patients. 3 patients underwent two cycles of neoadjuvant chemotherapy with Docetaxel combined with platinum. Thoracoscopy combined with laparoscopic resection was performed in 4 patients and mediastinoscopy combined with laparoscopic surgery in 3 patients. The posterior mediastinal esophageal bed pathway was used in all the patients. Moreover, all the patients undergoing surgery are squamous cell carcinoma.

The BPP repair was performed 35-60 days (median, 45 days) after radical operation of esophageal squamous cancer. The operative duration was 60-90 min (median, $75 \mathrm{~min}$ ). Oral intake was initiated 5-8 days (median, 6 days) after BPP repair process. No patients had dysphagia. No patients developed surgical site infection. No sign of recurrence anastomotic leakage was found after BPP repair in each patient.

\section{Discussion}

Cervical anastomotic leakage is one of the most common complications after esophageal cancer surgery. The incidence of anastomotic leakage after the esophagus reconstruction was as high as $20-25 \%$ (7). Tubular gastric is the common alternative organ after resection of esophageal squamous cell carcinoma. Either it is manual or mechanical anastomosis depends on surgeons' preferences and technology to some extent. Related studies showed that there was no significant difference in the incidence of an anastomotic fistula between mechanical and handsewn anastomotic techniques (8). Compared with cervical esophagogastric anastomosis, intrathoracic anastomosis is associated with higher complications and mortality. The incidence of cervical anastomotic leakage is higher than that of intrathoracic anastomotic leakage (9). The main reason for this difference is the longer distance that the blood 

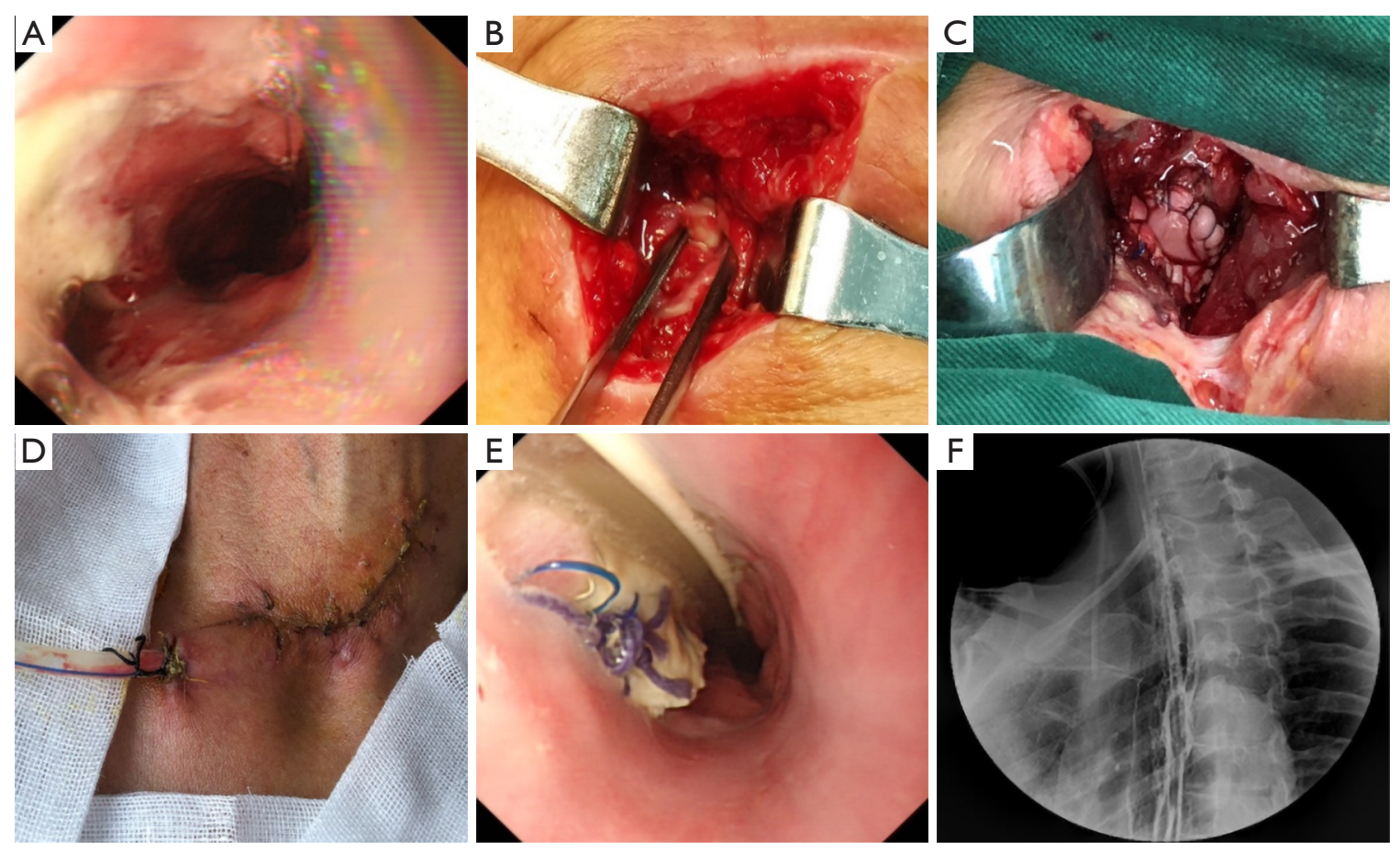

Figure 1 BPP repair and follow-up of cervical anastomotic leakage. (A) Postoperative endoscopy confirmed the neck anastomotic leak; (B) intraoperative exposure of the neck anastomotic leak position; (C) the position after repair the neck anastomotic leak in the second operation; (D) the state of the neck incision healed after the anastomotic leak repaired; (E) review the postoperative endoscopy after repairing the anastomotic leak; (F) review the postoperative upper gastrointestinal tract after repairing the anastomotic leak. BPP, bovine pericardium patch.

Table 1 Clinical features of patients undergoing BPP repair

\begin{tabular}{|c|c|c|c|c|c|c|c|c|c|c|c|}
\hline \multirow[b]{2}{*}{$\begin{array}{l}\text { Case } \\
\text { No. }\end{array}$} & \multicolumn{4}{|c|}{ Patients' characteristics } & \multicolumn{2}{|c|}{ Esophageal reconstruction } & \multirow{2}{*}{$\begin{array}{c}\text { Diagnosis of } \\
\text { anastomotic leakage } \\
\text { after esophagectomy, } \\
\text { days }\end{array}$} & \multicolumn{4}{|c|}{ Bovine pericardial patch repair } \\
\hline & $\begin{array}{l}\text { Age, } \\
\text { years }\end{array}$ & Sex & pTNM & NCT & MIE & Reconstruction route & & $\begin{array}{l}\text { BPP repair, } \\
\text { days }\end{array}$ & $\begin{array}{c}\text { duration of } \\
\text { operation, } \min \end{array}$ & $\begin{array}{l}\text { Relapse of } \\
\text { leakage }\end{array}$ & $\begin{array}{l}\text { Oral intake } \\
\text { after repair, days }\end{array}$ \\
\hline 1 & 55 & Male & IIIA & Yes & TLC & Posterior mediastinal & 5 & 37 & 63 & No & 5 \\
\hline 2 & 60 & Female & IIIB & Yes & TLC & Posterior mediastinal & 4 & 60 & 78 & No & 6 \\
\hline 4 & 67 & Male & IB & No & MLC & Posterior mediastinal & 6 & 35 & 60 & No & 7 \\
\hline 5 & 72 & Male & $\| A$ & No & TLC & Posterior mediastinal & 9 & 45 & 78 & No & 5 \\
\hline 6 & 77 & Male & IB & No & MLC & Posterior mediastinal & 10 & 50 & 90 & No & 8 \\
\hline 7 & 78 & Male & $\| A$ & No & MLC & Posterior mediastinal & 7 & 47 & 82 & No & 7 \\
\hline
\end{tabular}

NCT, neoadjuvant chemotherapy; TLS, thoracoscopy combined with laparoscopy; MLS, mediastinoscopy combined with laparoscopy; BPP, bovine pericardium patch; MIE, minimally invasive esophagus. 
supply needs to travel for anastomotic healing in the neck than in the intrathoracic region (10). In our department, end-to-side mechanical anastomosis is our first choice, and the embedded cutting edge is then reinforced with an absorbable suture.

In this study, $12.7 \%$ of the patients who underwent cervical mechanical anastomosis after esophagectomy developed anastomotic leakage. Leakage was confirmed in these patients by angiography or endoscopy. The three main factors of anastomotic leakage include reduced blood supply, anastomotic tension, and lack of serosa structure (11). Further risk factors are represented by immunosuppression, poor nutrition, and earlier radiation treatment (12). Consistent with most earlier studies, in the end-to-side anastomosis, the blood supply of the gastric stump is damaged in this study.

Once cervical anastomotic leakage occurs after the operation of esophageal squamous cell carcinoma, active anti-infection and drainage treatment is usually given first (13). Leakage is normally healed by granulation tissue filling, and Intractable anastomotic leakage is rare. For intractable cervical anastomotic leakage, active surgical intervention, or interventional treatment often need considering, such as vascularized pedicle tissue flaps, sternocleidomastoid flap repair, and covered stents most commonly used (14). As we know, the treatment of cervical anastomotic fistula with covered stents usually lead to severe swallowing discomfort or stent displacement (15). In clinical practice, vascularized pedicle tissue flaps can be used to repair cervical anastomotic fistula or to reinforce gastrointestinal anastomosis (16). Because of its rich muscle content, pectoralis major muscle is an ideal choice for the repair of anastomotic leakage. The sternocleidomastoid muscle can be transplanted in the same field for the repair of cervical anastomotic fistula. Compared with the pectoralis major muscle flap, the extent of the sternocleidomastoid flap translocation is relatively minimal (17). In contrast, the BPP is used for anastomotic fistula repair, and its operation is minimally invasive. Also, there have been few reports in earlier literature.

BPP is mainly used for repair of cardiovascular surgery. To prevent anastomotic dehiscence, there are reports in the literature where the use of biological materials has been investigated to reinforce an anastomosis (18). In an earlier experimental study in pigs, they conducted a comparative study using BPP embedding anastomosis. The results showed that the anastomotic healing after BPP embedding was better, and it was surprising in the prevention and treatment of peritonitis caused by anastomotic leakage (19). The knowledge derived from the experimental setting has been applied to esophageal cancer surgery in this study. Independently from the type and the anatomical level of anastomosis, when esophagogastric anastomoses have been wrapped with the patch, neither leak, stenosis, and abscess were seen, nor any cases of displacement and shrinkage of the BPP during follow-up. BPP is a kind of membrane with specific strengths and extensibilities, which is composed of multidirectional fibers and is completely degraded in the host (20). BPP can adequately cover fragile and vulnerable tissue and prevent exosmosis of digestive juice. Based on the previous evidence from animal studies, BPP may have ideal qualities as a tissue to use as anastomotic reinforcement, such as the trend for neoangiogenic function, reduction in adhesion formation, resistance to infection, and biocompatibility (21).

In this study, all leakage sites occurred at the anastomotic site. The debridement of necrotic tissue and the exposure of fistula in the cervical esophagogastric anastomotic area are essential for reliable repair (22). We performed BPP repair in these patients with refractory anastomotic fistula under general anesthesia. With regards to the choice of timing for the repair of anastomotic leakage, Hayashi et al. recommended that it be performed four weeks after the first operation because the anastomotic leakage was more likely to recur within three weeks (23). In our study, we recommend early debridement around the anastomotic area. Concerning the radical resection of esophageal squamous cell carcinoma method, double endoscopic esophagectomy with cervical anastomosis was performed. Cervical anastomosis is performed because intrathoracic anastomosis often leads to fatal clinical outcomes (24). Even if these results seem to be encouraging, but the efficacy of BPP for giant anastomotic leakage is unclear. Further investigation of the appropriateness of the BPP for giant anastomotic leakage is needed, and randomized controlled clinical trials are needed to compare the outcomes of anastomosis performed with and without the BPP.

In conclusion, BPP repair is a safe and effective processing scheme for patients with cervical anastomotic fistula after resection of esophageal squamous cell carcinoma. This method may be recommended for appropriate patients with intractable cervical anastomotic fistula.

\section{Acknowledgments}

None. 


\section{Footnote}

Conflicts of Interest: The authors have no conflicts of interest to declare.

Ethical Statement: The authors are accountable for all aspects of the work in ensuring that questions related to the accuracy or integrity of any part of the work are appropriately investigated and resolved. In accordance with the requirements of the Helsinki Declaration and relevant laws and regulations, such as the ethical review of biomedical research involving human beings, there is no conflict of interest in the study, which has been examined and approved by the Ethics Committee (Ethics Committee of Henan chest Hospital, 20175021). The authors are accountable for all aspects of the work in ensuring that questions related to the accuracy or integrity of any part of the work are appropriately investigated and resolved.

\section{References}

1. Booka E, Takeuchi H, Kikuchi H, et al. Recent advances in thoracoscopic esophagectomy for esophageal cancer. Asian J Endosc Surg 2019;12:19-29.

2. Kikuchi H, Takeuchi H. Future Perspectives of Surgery for Esophageal Cancer. Ann Thorac Cardiovasc Surg 2018;24:219-22.

3. Schoppmann SF, Prager G, Langer FB, et al. Open versus minimally invasive esophagectomy: a single-center case controlled study. Surg Endosc 2010;24:3044-53.

4. Aurello P, Berardi G, Moschetta G, et al. Surgery for Carcinoma of the Distal Esophagus and Gastroesophageal Junction: A Systematic Review. Anticancer Res 2019;39:1651-60.

5. Han H, Pan M, Tao Y, et al. Early Enteral Nutrition is Associated with Faster Post-Esophagectomy Recovery in Chinese Esophageal Cancer Patients: A Retrospective Cohort Study. Nutr Cancer 2018;70:221-8.

6. Choi HK, Law S, Chu KM, et al. The value of neck drain in esophageal surgery: a randomized trial. Dis Esophagus 2017;11:40-2.

7. Saeki H, Tsutsumi S, Tajiri H, et al. Prognostic Significance of Postoperative Complications After Curative Resection for Patients With Esophageal Squamous Cell Carcinoma. Ann Surg 2017;265:527-33.

8. Rostas JW, Graffree BD, Scoggins CR, et al. Long-term outcomes after hand-sewn versus circular-stapled (25 and $29 \mathrm{~mm}$ ) anastomotic technique after esophagogastrectomy for esophageal cancer. J Surg Oncol 2018;117:469-72.

9. Gooszen JAH, Goense L, Gisbertz SS, et al. Intrathoracic versus cervical anastomosis and predictors of anastomotic leakage after oesophagectomy for cancer. Br J Surg 2018;105:552-60.

10. Biere SS, Maas KW, Cuesta MA, et al. Cervical or thoracic anastomosis after esophagectomy for cancer: a systematic review and meta-analysis. Dig Surg 2011;28:29-35.

11. Walther B, Johansson J, Johnsson F, et al. Cervical or thoracic anastomosis after esophageal resection and gastric tube reconstruction: a prospective randomized trial comparing sutured neck anastomosis with stapled intrathoracic anastomosis. Ann Surg 2003;238:803-12.

12. Goense L, Meziani J, Ruurda JP, et al. Impact of postoperative complications on outcomes after oesophagectomy for cancer. Br J Surg 2019;106:111-9.

13. Virgilio E, Ceci D, Cavallini M, et al. Surgical Endoscopic Vacuum-assisted Closure Therapy (EVAC)in Treating Anastomotic Leakages After Major Resective Surgery of Esophageal and Gastric Cancer. Anticancer Res 2018;38:5581-7.

14. Hagens ERC, Anderegg MCJ, Gisbertz SS, et al. International Survey on the Management of Anastomotic Leakage After Esophageal Resection. Ann Thorac Surg 2018;106:1702-8.

15. Valli PV, Mertens JC, Kröger A, et al. Stent-over-sponge (SOS): a novel technique complementing endosponge therapy for foregut leaks and perforations. Endoscopy 2018;50:148-53.

16. El-Negery A, Elmoghazy NA, Elgeidi A, et al. Vascularized fibular medialization for reconstruction of the tibial defects following tumour excision. Int Orthop 2017;41:2179-87.

17. Nakajima M, Satomura H, Takahashi M, et al. Effectiveness of Sternocleidomastoid Flap Repair for Cervical Anastomotic Leakage after Esophageal Reconstruction. Dig Surg 2014;31:306-11.

18. Noma K, Shirakawa Y, Kanaya N, et al. Visualized Evaluation of Blood Flow to the Gastric Conduit and Complications in Esophageal Reconstruction. J Am Coll Surg 2018;226:241-51.

19. Testini $M$, Gurrado A, Portincasa P, et al. Bovine pericardium patch wrapping intestinal anastomosis improves healing process and prevents leakage in a pig model. PLoS One 2014;9:e86627.

20. Gurrado A, Franco IF, Lissidini G, et al. Impact of pericardium bovine patch on incisional hernia treatment in contaminated or potentially contaminated fields: retrospective comparative study. Hernia 2015;19:259-66. 
21. Gurrado A, D'Ambra L, Sgaramella LI, et al. A Novel Technique of Digestive Anastomosis Reinforcement Using Bovine Pericardium Patch. Am Surg 2019;85:e79-e82.

22. Liu QX, Deng XF, Wang JS, et al. Use of oesophageal flap valvuloplasty and wrapping suturing technique in preventing postoperative complications after oesophagectomy for oesophageal cancer. Eur J Surg Oncol 2014;40:1355-60.

23. Hayashi K, Watanabe H, Kato H, et al. A clinical study of

Cite this article as: Hua X, Qian R, Shi K, Wei X, Zhang H, Qu G, Chen M, Zhang B. Effectiveness and safety of bovine pericardiumpatch repair for cervical anastomotic leakage after oesophagectomy for cancer. J Thorac Dis 2019;11(9):38083813. doi: $10.21037 /$ jtd.2019.09.12 the reanastomotic cases with thoracic esophageal cancer after resection and reconstruction with special reference to anastomotic method and results. Jpn J Gastroenterol Surg 1990;23:2315-21.

24. Kukar M, Ben-David K, Peng JS, et al. Minimally Invasive Ivor Lewis Esophagectomy with Linear Stapled Anastomosis Associated with Low Leak and Stricture Rates. J Gastrointest Surg 2019. [Epub ahead of print]. 